\title{
Correlation of endoscopic findings with Doppler ultrasound in portal hypertension in children
}

\author{
Swati Singh, Rasika Bhamre, Naman Shetty, Himali Meshram, Saumil Shah, Ira Shah \\ Department of Pediatric Gastroenterolgy and Hepatology, Bai Jerbai Wadia Hospital of Children, Mumbai, India
}

\begin{abstract}
Aim of the study: To determine the correlation of the endoscopic findings with portal Doppler and ultrasound (USG) in children with suspected portal hypertension (PHT).

Material and methods: Eighty children with extrahepatic portal vein obstruction (EHPVO) and chronic liver disease (CLD) were included in this retrospective study conducted over a period of 1 year. All patients underwent upper gastrointestinal (GI) endoscopy and Doppler.

Results: The etiology was EHPVO in 30 (37.5\%) patients, biliary atresia in 12 (15\%), Budd-Chiari syndrome in $11(13.7 \%)$, Wilson's disease in $10(12.5 \%)$, idiopathic CLD in $8(10 \%)$, autoimmune hepatitis in $4(5 \%)$, glycogen storage disease (GSD) in $3(3.8 \%)$, non-alcoholic liver disease (NAFLD) in $1(1.3 \%)$ and systemic lupus erythematosus (SLE) in $1(1.3 \%)$ patient. Fifty-three (66.25\%) patients had esophageal varices on endoscopy, of whom $3(3.8 \%)$ had associated gastric varices. Portal hypertensive gastropathy (PHG) was present in $30(37.5 \%)$ patients, of whom 10 (12.5\%) had severe PHG. Forty-one (51.3\%) patients had PHT on Doppler ( $\kappa$ correlation 0.43 ). Kappa correlation was 0.43 in patients with biliary atresia, 0.31 in Budd-Chiari syndrome, 0.23 in idiopathic CLD, 0.21 in CLD, and 0.05 in Wilson's disease. All (100\%) EHPVO patients and 39 (78\%) CLD patients had PHT on USG. Endoscopic findings of PHT were seen in 24 (80\%) EHPVO patients and 29 (58\%) CLD patients. All patients with EHPVO had cavernous transformation of the portal vein on Doppler. For patients with CLD, the common Doppler findings were collaterals seen in 35 patients and reversal of flow in 12 patients. Conclusions: Doppler ultrasound followed by endoscopy should be used to diagnose PHT in children. In children with biliary atresia, Doppler ultrasound may miss changes of PHT.
\end{abstract}

Key words: children, PHT, EHPVO, upper endoscopy, CLD.

Address for correspondence:

Ira Shah, Department of Pediatric Gastroenterology and Hepatology, Bai Jerbai Wadia Hospital for Children, Acharya Donde Marg, Parel, Mumbai, Maharashtra, India, 400 012, e-mail: irashah@pediatriconcall.com

\section{Introduction}

A variety of liver disorders, which may lead to portal hypertension, are encountered in children which are distinct from adults. Normal portal venous pressure is $7 \mathrm{mmHg}$. Hepatic venous portal gradient (HVPG) is defined as portal pressure above $10-12 \mathrm{~mm}$ of $\mathrm{Hg}$ $[1,2]$. HVPG results from a combination of increased portal blood flow and increased portal resistance [1]. In Indian children, variceal bleeding has been found to be the foremost reason for upper gastrointestinal (GI) bleeding compared to peptic ulcer in the western world [2]. In the Indian scenario, extrahepatic portal vein obstruction (EHPVO) is the cause of portal hypertension (PHT) in $68-76 \%$ cases. The other common causes are cirrhosis (24-28\%), non-cirrhotic portal fibrosis (4\%) and Budd-Chiari syndrome (3\%) [2]. PHT results in portosystemic communicating venous channels at numerous sites giving rise to oesophageal, gastric and colonic varices [2]. PHT manifests with complications such as varices or portal hypertensive gastropathy. Very often, the two conditions occur concomitantly. 
The endoscopic appearance of portal hypertensive gastric mucosa includes several lesions such as: fine pink speckling, scarlatina-type rash petechia, multiple bleeding spots, papules, superficial reddening, snakeskin pattern, cherry red spots and mosaic-like pattern [3]. As the portal hypertensive gastropathy (PHG) becomes more severe, the mucosa becomes friable and bleeds on contact. Frequently, hemorrhagic spots are seen on the gastric mucosa. Typically, PHG is most evident in the proximal stomach. Clinically, varices present with acute onset of upper GI bleeding which might be torrential and life-threatening because of high pressure in the distended veins $[3,4]$. Patients with PHG also present with upper GI bleeding.

Ultrasound (USG) with Doppler is the first-line imaging examination to be performed in patients with suspected liver disease and/or PHT. It is safe, easily repeatable, not very expensive and very sensitive in detecting thrombosis in the portal vein and hepatic veins. These features enable the formation of correct differential diagnoses in new cases of PHT [5-7]. Only two signs are $100 \%$ specific signs of PHT: porto-systemic collaterals (e.g. paraumbilical vein, spleno-renal collaterals, etc.) and reversal of flow within the portal vein system. Splenomegaly is often related to PHT; this sign is more sensitive than other signs but less specific. However, increasing spleen size is an independent predictor of gastroesophageal varices in compensated cirrhosis cases [6]. Other signs include dilatation of the portal venous system vessels, lack of or reduced respiratory variations of splenic and superior mesenteric vein diameter, reduced portal vein velocity, increased congestion index of the portal vein and an altered Doppler pattern in the liver veins $[7,8]$. Less commonly explored signs include changes in the arterial flow pattern of the hepatic, splenic, mesenteric and renal arteries. As most of these signs show some degree of correlation with the HVPG, none of them can be used as a reliable surrogate for hemodynamic measurement either at first examination or after starting non-selective $\beta$-blocker therapy $[9,10]$. Hence, we conducted this study to determine the correlation of the endoscopic findings with portal Doppler and USG in children with suspected PHT.

\section{Material and methods}

Eighty children who underwent endoscopy were included in the study. This retrospective study was conducted over a period of 1 year from June 2018 to 2019 after approval from the hospital ethics committee. Patients with chronic liver disease (CLD) or EHPVO were included in the study [11]. PHT on Doppler was diagnosed if there was reversal of flow, presence of gastric or splenic collaterals or presence of portal cavernoma [6].

Doppler ultrasound was done before endoscopy. Most of the patients had USG examination earlier also and were known to have PHT.

All endoscopies were performed using an Olympus GIF-H170 Optera video gastrointestinal scope. Endoscopic features of portal hypertension such as presence of esophageal varices, number of varices, grade of varices [12] and presence of fundic varices were noted. Mild PHG was diagnosed if there was presence of snake skin pattern in the stomach and severe PHG was associated with erosions [13].

Laboratory and demographic data at the time of endoscopy such as age, gender, etiology of CLD, liver function tests, hemogram, and Child-Pugh score were noted. Thrombocytopenia was determined if the platelet count was $<150,000$ cells $/ \mathrm{mm}^{3}$.

Aspartate aminotransferase (AST) to platelet ratio index (APRI) score was calculated using the following formula:

APRI score $=[($ AST/upper normal limit of AST $)$ $\times 100] /$ platelet count

AST was measured in IU/l, the upper normal limit was taken as $40 \mathrm{IU} / \mathrm{l}$. The platelet count was measured in $=10^{9} / 1$.

\section{Statistical analysis}

Prevalence of PHT on endoscopy and Doppler ultrasound was determined. Kappa correlation was used to determine the agreement between PHT detected on endoscopy and that with Doppler in various etiologies of PHT. Kappa $<0$ was considered as less than chance agreement, 0.01-0.20 slight agreement, 0.21-0.40 fair agreement, 0.41-0.60 moderate agreement, 0.61-0.80 substantial agreement and 0.81-0.99 almost perfect agreement.

\section{Results}

The mean age at the time of endoscopy was 7.38 \pm 4.17 years with a range of $1-18$ years. Male : female ratio was $51: 29$. The etiology was EHPVO in $30(37.5 \%)$ patients, biliary atresia in $12(15 \%)$, BuddChiari syndrome in 11 (13.7\%), Wilson's disease in $10(12.5 \%)$, idiopathic CLD in $8(10 \%)$, autoimmune hepatitis in $4(5 \%)$, glycogen storage disease (GSD) in $3(3.8 \%)$, non-alcoholic liver disease (NAFLD) in $1(1.3 \%)$ and systemic lupus erythematosus (SLE) in $1(1.3 \%)$ patient. Previous hematemesis was noted in $19(23.7 \%)$ patients and $13(16.3 \%)$ had melena. 
Forty-one (51.2\%) patients had thrombocytopenia. The mean hemoglobin was $10.32 \pm 1.32 \mathrm{gm} / \mathrm{dl}$, white cell count was $7.58 \pm 4.54$ cells $/ \mathrm{mm}^{3}$ and platelets of $1.82 \pm 1.09$ cells $/ \mathrm{mm}^{3}$. Mean serum glutamic-oxaloacetic transaminase (SGOT) was $89.62 \pm 193.79 \mathrm{IU} / 1$ and serum glutamic pyruvic transaminase (SGPT) was $70.10 \pm 147.57 \mathrm{IU} / \mathrm{l}$. Splenomegaly was present in $62(77.5 \%)$ patients. The mean splenic size on USG was $70.10 \pm 147.57 \mathrm{~mm}$. On Doppler, 41 (51.3\%) had changes of PHT. All (100\%) EHPVO patients and 39 (78\%) CLD patients had PHT on USG. Endoscopic findings of PHT were seen in 24 (80\%) EHPVO patients and 29 (58\%) CLD patients. All patients with EHPVO had cavernous transformation of the portal vein on Doppler. For patients with CLD, the common Doppler findings were collaterals seen in 35 patients and reversal of flow in 12 patients. Fifty-three (66.25\%) patients had esophageal varices on endoscopy ( $\kappa$ correlation 0.43$)$, of whom $3(3.8 \%)$ had associated gastric varices. PHG was present in $30(37.5 \%)$ patients, of whom 19 (23.7\%) had mild PHG, and 1 (1.3\%) and 10 (12.5\%) had severe PHG. Correlation of endoscopic findings and Doppler ultrasound is depicted in Table 1. Table 2 compares factors associated with endoscopic PHT and no endoscopic PHT. The platelet count $(p=0.06)$ and APRI index $(p=0.031)$ were significant-

Table 1. Correlation of portal hypertension (PHT) as detected by ultrasound (USG) and endoscopy according to diagnosis

\begin{tabular}{lcccc}
\hline Diagnosis & $\boldsymbol{n}$ & $\begin{array}{c}\text { USG PHT } \\
\boldsymbol{n}(\%)\end{array}$ & $\begin{array}{c}\text { Endoscopic PHT } \\
\boldsymbol{n}(\%)\end{array}$ & $\kappa$ correlation \\
\hline EHPVO & 30 & $30(100.0)$ & $24(80.0)$ & NA \\
\hline CLD & 50 & $39(78.0)$ & $29(58.0)$ & 0.21 (fair) \\
\hline Biliary atresia & 12 & $9(75.0)$ & $11(91.7)$ & 0.43 (moderate) \\
\hline $\begin{array}{l}\text { Budd-Chiari } \\
\text { syndrome }\end{array}$ & 11 & $9(81.8)$ & $5(45.5)$ & 0.31 (fair) \\
\hline Wilson syndrome & 10 & $8(80.0)$ & $3(30.0)$ & 0.05 (slight) \\
\hline Idiopathic CLD & 8 & $7(87.5)$ & $5(62.5)$ & 0.23 (fair) \\
\hline CLD - chronic liver disease & & &
\end{tabular}

ly lower in patients with endoscopic PHT. Factors associated with CLD and EHPVO are analyzed in Table 3.

\section{Discussion}

Whenever there is associated liver disease, the course of portal hypertension per se becomes more complicated due to signs of liver failure such as jaundice, ascites, encephalopathy, etc. along with the usual GI bleeding. At times, patients may present without evidence of portal hypertension. Hence, such patients should undergo diagnostic endoscopies to look for varices [14]. The Doppler ultrasound did not detect PHT in patients with biliary atresia, which was subsequently detected on endoscopy. The risk of portal hypertension in biliary atresia patients is high in the early years of life [15]. Hence, diagnostic endoscopy should be performed in these patients. Due to the rarity of spontaneous regression of varices, primary prophylaxis for bleeding is indicated in patients with gastric varices along the cardia, esophageal varices and red markings [15]. Portal hypertension was seldom seen in patients with Wilson's disease in our study and hence Doppler and upper GI endoscopy were equivalent.

Extrahepatic portal vein obstruction is defined as the obstruction of the extrahepatic portal vein with

Table 2. Comparison of endoscopic portal hypertension (PHT) with no endoscopic PHT

\begin{tabular}{lccc}
\hline Variable & $\begin{array}{c}\text { Endoscopic PHT } \\
\text { (varices and/or portal } \\
\text { HT gastropathy) } \\
(\boldsymbol{n}=53)\end{array}$ & $\begin{array}{c}\text { No PHT on } \\
\text { endoscopy } \\
(\boldsymbol{n}=27)\end{array}$ & $P$ value \\
\hline Platelet count & $1.75 \pm 1.50$ & $2.21 \pm 1.30$ & 0.06 \\
\hline Thrombocytopenia & $30(56.6 \%)$ & $11(42.3 \%)$ & 0.24 \\
\hline APRI score & $1.11 \pm 1.03$ & $2.70 \pm 5.99$ & 0.031 \\
\hline Splenomegaly & $50(94.3 \%)$ & $12(44.4 \%)$ & $<0.00001$ \\
\hline
\end{tabular}

Table 3. Factors associated with chronic liver disease and extrahepatic portal vein obstruction (EHPVO)

\begin{tabular}{|c|c|c|c|}
\hline Factors & Chronic liver disease $(n=50)$ & $\operatorname{EHPVO}(n=30)$ & $P$ value \\
\hline \multicolumn{4}{|l|}{ Ultrasound findings } \\
\hline Reversal of flow & $12(24 \%)$ & $4(13.3 \%)$ & 0.387 \\
\hline Gastric or splenic collaterals & $35(70 \%)$ & $26(86.7 \%)$ & 0.110 \\
\hline Thrombocytopenia & $25(50 \%)$ & $16(53.3 \%)$ & 0.820 \\
\hline Previous hematemesis (no. of episodes) & $4.32 \pm 2.77$ & $5 \pm 0$ & 0.092 \\
\hline Previous melena & $6(12 \%)$ & $13(43.3 \%)$ & 0.002 \\
\hline Splenomegaly & $38(76 \%)$ & $26(86.7 \%)$ & 0.171 \\
\hline Platelet count $\left(\times 10^{9}\right.$ cells $\left./ \mathrm{mm}^{3}\right)$ & $1.97 \pm 1.35$ & $1.79 \pm 1.10$ & 0.263 \\
\hline
\end{tabular}


or without the involvement of the intrahepatic portal veins. It is characterized by features of recent thrombosis or portal hypertension with portal cavernoma as a sequela of portal vein obstruction [11]. Usually, Doppler ultrasonography is the first imaging modality used and is accurate in the assessment of the portal venous system [16]. EHPVO in childhood is most often chronic and presents with features of variceal bleeding and splenomegaly [16]. Initial thrombus formation is asymptomatic and the only sign may be the formation of new vessels, which on Doppler ultrasonography is known as portal cavernoma. Thus, most patients with EHPVO will have collaterals on Doppler.

Endoscopy has a significantly higher sensitivity regarding the diagnosis of portal hypertension and permits visualization of collaterals belonging to intrinsic (deep) venous circulation, which if large can significantly increase the risk of variceal bleeding. Also, therapeutic intervention can be done at the time of endoscopy. Children with thrombocytopenia and splenomegaly can be considered for surveillance endoscopy with an intention to treat using sclerotherapy or band ligation [17]. Patients with low platelet counts and low APRI scores are more likely to have findings of PHT on endoscopy. Unfortunately, it is an invasive procedure and remains not widely available [18-20]. On the other hand, Doppler ultrasound is a non-invasive method that provides precise information regarding blood flow in major vessels of the abdomen [21]. However, it remains non-therapeutic. Plestina et al. [22] suggested that Doppler ultrasound may be of use in prediction of the risk for oesophageal variceal bleeding. However, these findings are inconsistent with the results of other studies. For example, Berzigotti et al. [23] found that color Doppler ultrasound played no role in predicting clinically significant portal hypertension and esophageal varices. Also, Cioni et al. [24] found no relationship between the parameters of portal flow and the risk of bleeding, while Li et al. reported that Doppler ultrasound parameters of the portal vein exhibited no correlation with the severity of endoscopic abnormalities in patients with cirrhosis [21].

\section{Conclusions}

Both endoscopy and Doppler ultrasonography are needed to best diagnose and for appropriate management of portal hypertension. Doppler ultrasonography, being non-invasive, should be used first, after which detailed evaluation can be carried out by endoscopy. It is extremely useful in patients with EHPVO. However, in children with biliary atresia, endoscopy may be performed even if the Doppler does not show changes of PHT.

\section{Limitations}

Most patients were known to have PHT as they had undergone US examination earlier. This could have promoted observer bias.

\section{Disclosure}

The authors declare no conflict of interest.

\section{References}

1. Loening-Baucke V. Chronic constipation in children. Gastroenterology 1993; 105: 1557-1564.

2. Kuizenga WA, Benninga MA. Functional constipation and incontinence. In: Wyllie R, Hyams J, Kay M (Eds.). Pediatric gastrointestinal and liver disease. 5th ed. Elsevier, USA 2016; 124-136.

3. Mugie SM, Benninga MA, Di Lorenzo C. Epidemiology of constipation in children and adults: a systematic review. Best Pract Res Clin Gastroenterol 2011; 25: 3-18.

4. Gupte S, Anderson RA. Constipation. In: Gupte S (Ed.). The Short Textbook of Pediatrics. 11th ed. Jaypee, New Delhi 2009; 472-473.

5. Schiller LR. Colonic symptoms, constipation, diarrhea and fecal incontinence. In: Rao SSC, Parkman HP, McCallum RW (Eds.). Handbook of gastrointestinal motility and functional disorders. SLACK incorporated, USA 2015; 211-219.

6. Tabbers MM, DiLorenzo C, Berger MY, et al. Evaluation and treatment of functional constipation in infants and children: evidence-based recommendations from ESPGHAN and NASPGHAN. J Pediatr Gastroenterol Nutr 2014; 58: 258-274.

7. Levy El, Lemmens R, Vandenplas Y, Devreker T. Functional constipation in children: challenges and solutions. Pediatric Health Med Ther 2017; 8: 19-27.

8. Rodriguez L, Sood M, Di Lorenzo C, Saps M. An ANMS-NASPGHAN consensus document on anorectal and colonic manometry in children. Neurogastroenterol Motil 2017; 29: e12944.

9. Rodriguez L, Nurko S. Gastrointestinal motility procedure. In: Wyllie R, Hyams J, Kay M (Eds.). Pediatric gastrointestinal and liver disease. 5th ed. Elsevier, USA 2016; 748-765.

10. Carrington EV. Studies of anorectal functioning using high resolution anorectal manometry in health and fecal incontinence. The Blizard Institute of Cell and Molecular Sciences, Bart and the London School of Medicine and Dentistry, Queen Mary, University of London 2016.

11. De FR, Faculty BV. Revising consensus in portal hypertension: Report of the Baveno V consensus workshop on methodology of diagnosis and therapy in portal hypertension. J Hepatol 2010; 53: $762-768$.

12. Paquet KJ. Prophylactic endoscopic sclerosing treatment of the esophageal wall in varices - a prospective controlled randomized trial. Endoscopy 1982;14: 4-5.

13. Urrunaga NH, Rockey DC. Portal hypertensives gastropathy and colopathy. Clin Liver Dis 2014; 8: 389-406.

14. Mahajan AJ, Ghildiyal RG, Karnik P. Clinicopathological correlation of portal hypertension in children and management strategies. Int J Biomed Adv Res 2018; 9: 70-75.

15. Duché M, Ducot B, Tournay E, et al. Prognostic value of endoscopy in children with biliary atresia at risk for early devel- 
opment of varices and bleeding. Gastroenterology 2010; 139: 1952-1960.

16. Achar S, Dutta HK, Gogoi RK. Extrahepatic portal vein obstruction in children: Role of preoperative imaging. J Indian Assoc Pediatr Surg 2017; 22: 144-149.

17. Grammatikopoulos T, McKiernan PJ, Dhawan A. Portal hypertension and its management in children. Arch Dis Child 2018; 103: 186-191.

18. Caletti GC, Brocchi E, Ferrari A, et al. Value of endoscopic ultrasonography in the management of portal hypertension. Endoscopy 1992; 24: 342-346.

19. DeAngelis C, Pellicano R, Carucci P, et al. Endoscopic ultrasonography in hepatology: focus on portal hypertension. Minerva Gastroenterol Dietol 2008; 54: 131-139.

20. Hino S, Kakutani H, Ikeda K, et al. Hemodynamic assessment of the left gastric vein in patients with esophageal varices with color Doppler EUS: factors affecting development of esophageal varices. Gastrointest Endosc 2002; 55: 512-517.

21. Li J, Hao FH, Xia JG, et al. Hemodynamic analysis of esophageal varices in patients with liver cirrhosis using color Doppler ultrasound. World J Gastroenterol 2005; 11: 4560-4565.

22. Plestina S, Pulanic R, Kralik M, et al. Color Doppler ultrasonography is reliable in assessing the risk of esophageal variceal bleeding in patients with liver cirrhosis. Wiener Klinische Wochenschrift 2005; 117: 711-717.

23. Berzigotti A, Gilabert R, Abraldes JG, et al. Noninvasive prediction of clinically significant portal hypertension and esophageal varices in patients with compensated liver cirrhosis. Am J Gastroenterol 2008; 103: 1159-1167.

24. Cioni G, Tincani E, Cristanietal A. Does the measurement of portal flow velocity have any value in the identification of patients with cirrhosis at risk of digestive bleeding? Liver 1996; 16: 84-87. 